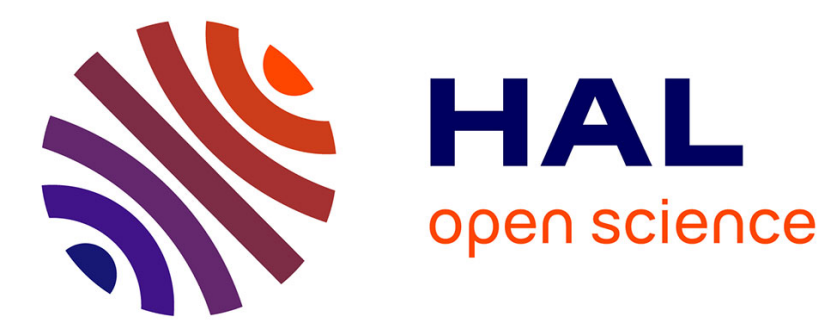

\title{
Stochastic resonance in an analog current-mode neuromorphic circuit
}

Damien Querlioz, Vincent Trauchessec

\section{To cite this version:}

Damien Querlioz, Vincent Trauchessec. Stochastic resonance in an analog current-mode neuromorphic circuit. 2013 IEEE International Symposium on Circuits and Systems (ISCAS), May 2013, Beijing, China. 10.1109/ISCAS.2013.6572166 . hal-01827053

\section{HAL Id: hal-01827053 https://hal.science/hal-01827053}

Submitted on 1 Jul 2018

HAL is a multi-disciplinary open access archive for the deposit and dissemination of scientific research documents, whether they are published or not. The documents may come from teaching and research institutions in France or abroad, or from public or private research centers.
L'archive ouverte pluridisciplinaire HAL, est destinée au dépôt et à la diffusion de documents scientifiques de niveau recherche, publiés ou non, émanant des établissements d'enseignement et de recherche français ou étrangers, des laboratoires publics ou privés. 


\title{
Stochastic Resonance in an Analog Current-Mode Neuromorphic Circuit
}

\author{
Damien Querlioz, Vincent Trauchessec \\ Institut d'Electronique Fondamentale, Univ. Paris-Sud, CNRS \\ Orsay, France \\ damien.querlioz@u-psud.fr
}

\begin{abstract}
Stochastic resonance is a general phenomenon by which the sensitivity of a system to small inputs may be increased by the addition of noise. In this paper, we show that a neuro-inspired analog circuit naturally exhibits stochastic resonance. Transient circuit simulations allow the recognition of the evidence of this phenomenon. Detailed analyses show the importance of well choosing a specific neuronal parameter, the refractory period, so that the resonance can be used in practice. These results open the way for neuromorphic designs to process noisy data without signal processing, or to work in extremely noisy environments.
\end{abstract}

Keywords: stochastic resonance, neuromorphic, spiking neural networks, noise.

\section{INTRODUCTION}

Noise and unpredictability are becoming central issues in electronics circuits and systems research. Many modern applications of electronics (biological, medical or ubiquitous sensing applications) have to process naturally noisy information from the real world, using a low power budget. Furthermore, microelectronics technology itself becomes noisier in advanced technologies [1]. This should be even more crucial if the channel material in CMOS switches to nanostructures like silicon nanowires, which exhibit high noise [2]. A higher noise tolerance could also allow reducing supply voltage, thus leading to power savings.

Electronic circuits that work like the brain with asynchronous spikes - neuromorphic circuits - have been developed since the late 80s [3], [4] and are known to operate well with real life natural data [5], [6]. It it thus natural to assess their potential for working in extremely noisy environments. This is all the more significant since it has been shown in Neuroscience works that biological neurons have extreme noise tolerance. They may even in some instances benefit from noise, exploiting an apparently paradoxical phenomenon known as stochastic resonance. This has been observed theoretically [7] and experimentally [8].

Stochastic resonance was originally introduced in the $80 \mathrm{~s}$ to explain climatologic cycles, and has been observed in various physical and biological systems [9], [10], and largely theorized [11]. It states that, in some situations, the response of a system to a small stimulus may be improved by noise, and that a noise optimum may exist. The goal of this paper is to analyze if a CMOS-based neuromorphic neuron can express stochastic resonance, and to analyze its behavior. For this purpose, we perform transient circuit simulations.

The idea of getting an electronic system to express stochastic resonance has previously been proposed using nanoelectronics devices: carbon nanotubes [12], single electron devices [13] and tunnel diodes with negative differential resistance [14-16]. It has also been proposed in bistable CMOS circuits [17], [18], which implement the traditional equations of stochastic resonance.

In this work, we propose to use a spiking neuromorphic CMOS circuit that can be fabricated with current commercial technologies. Unlike the previous proposals of stochastic resonant circuits, this kind of spiking design is developed widely and has been proven to scale to large systems [19-23]. Exploiting stochastic resonance could be a significant advance for this kind of circuits.

The paper is organized as follows. First, we introduce the circuit and its simulation methodology. Second, we present our results and identify the stochastic resonance. Third, we discuss its meaning and significance.

\section{CIRCUIT AND MethodS}

The circuit is the log-domain current-mode generalized integrate-and-fire neuron from [3], and presented in Figure 1. We stripped it from the adaptation sub-circuit, which was not necessary for this work. As many neuromorphic circuits [22], the circuit uses transistors operating in the subthreshold regime, and exploits the exponential dependence of current to gate voltage. As an output, it generates digital asynchronous spikes, which can be routed to other neurons, synapses or generic digital circuits. The neuron works in real time (meaning, with time scales similar to the ones of biological neurons), which makes it appropriate to process real world dynamic data in real time. 


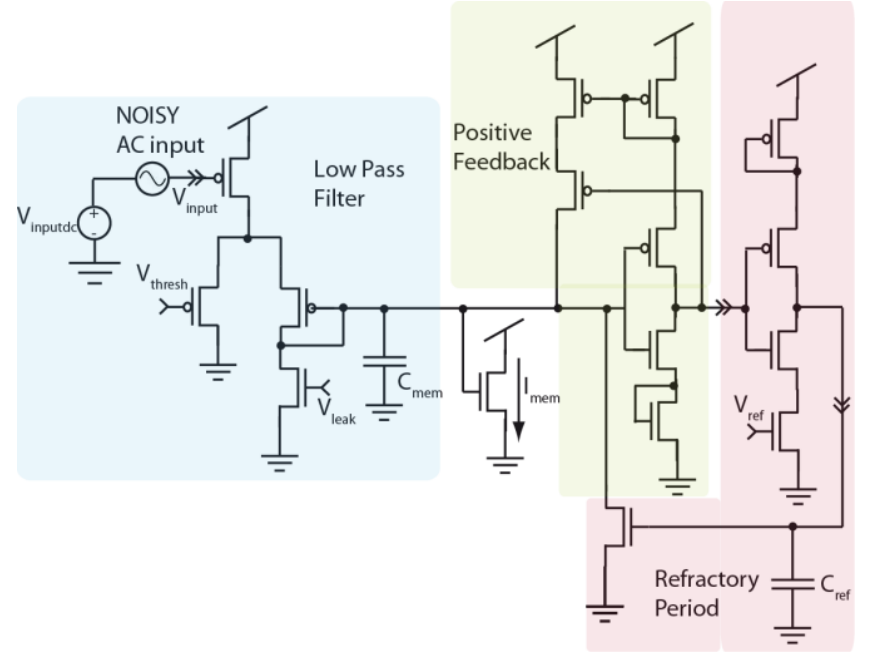

Figure 1. Neurons circuit used in this work (based on [3])

The circuit is built around a low pass filter block [24]. The input is applied as a voltage on the gate of a PMOS transistor. Another block implements a positive feedback. When $\mathrm{C}_{\mathrm{mem}}$ is charged enough, the positive feedback becomes dominant, which leads to the switch of the digital transistors: the neuron spikes (or "fires"). After the neuron has spiked, the refractory period block keeps $\mathrm{C}_{\mathrm{mem}}$ discharged for a given duration, preventing the neuron from firing again. The refractory period duration is controlled directly by the $\mathrm{V}_{\text {ref }}$ bias and the $\mathrm{C}_{\mathrm{ref}}$ capacitance value. All details and theory about the circuit are given in [3].

The input was a sine function (at $10 \mathrm{~Hz}$ in most of the paper). In all simulations, the amplitude of the input is small enough, so that when no noise is present, the neuron does not spike. Any spike is thus caused by noise.

Noise was introduced in the circuit with an artificial voltage noise source in series with the input. This noise source was a custom device programmed in the Verilog-A language, and generated white noise.

To design the circuit, we used the design kit of a $65 \mathrm{~nm}$ Low Power technology from a commercial vendor. The analog transistors were implemented with the $\mathrm{I} / \mathrm{O}$ transistors of the technology (regular transistors had too high leakage for an operation of the circuit in biological real time). The two capacitors $\mathrm{C}_{\mathrm{mem}}$ and $\mathrm{C}_{\mathrm{ref}}$ were also implemented with $\mathrm{I} / \mathrm{O}$ transistors.

The circuit was simulated with Cadence Spectre, in transient noise simulation, with various biases and $\mathrm{C}_{\mathrm{mem}}$ and $\mathrm{C}_{\text {ref }}$ capacitance values. Considering the low currents, and the permanent discontinuities introduced by noise, simulations required extremely strict tolerance criteria (vtol, itol, reltol, gmin) to ensure a reliable result. Simulation convergence was thus extremely slow, in comparison with traditional transient simulation of analog circuits. To initiate the circuit properly, the leakage bias $\mathrm{V}_{\text {leak }}$ was originally set at 0 volts and then rapidly increased to its final value.
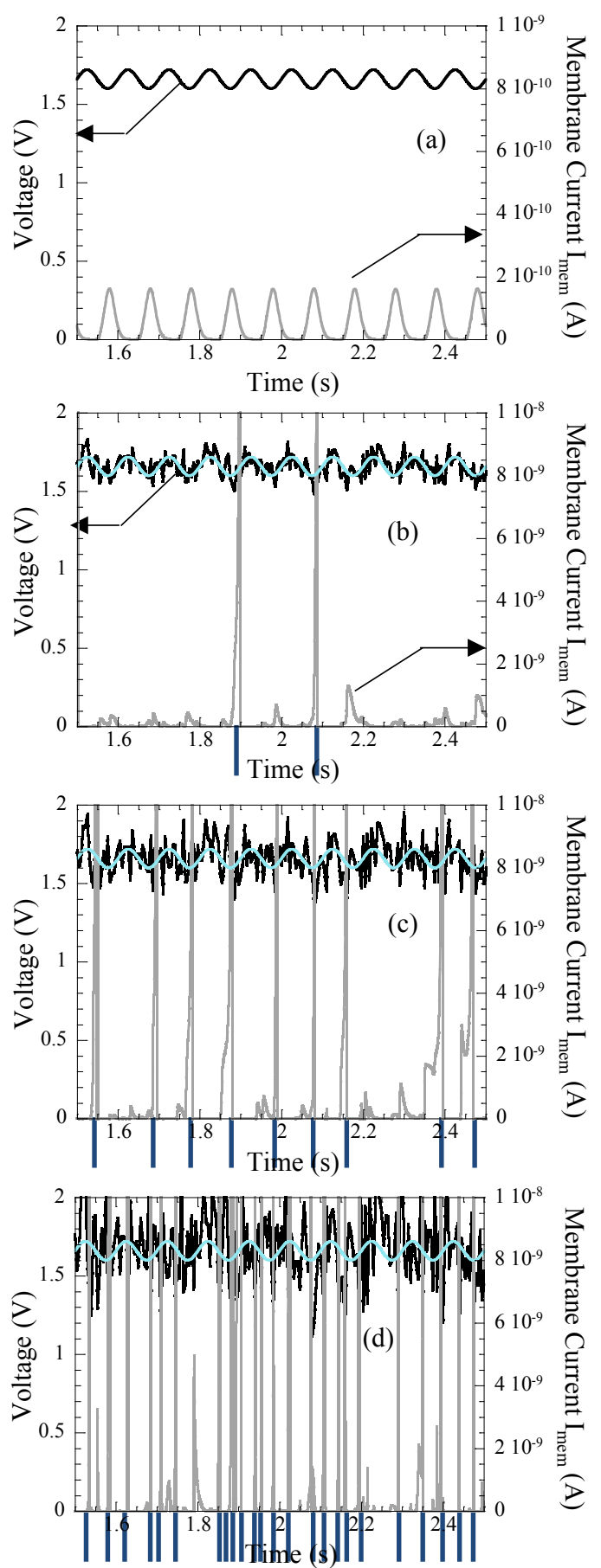

Figure 2. For different noise levels, input voltage $\mathrm{V}_{\text {input }}$ (black), small AC input without the noise (light blue), membrane current $I_{\text {mem }}$ (grey). Neurons spikes are materialized by the vertical blue lines on the time axis. (a) No input noise (b) $0.04 \mathrm{~V}^{2} / \mathrm{kHz}$ (c) $0.1 \mathrm{~V}^{2} / \mathrm{kHz}$ (d) $0.6 \mathrm{~V}^{2} / \mathrm{kHz}$

The circuit was simulated during $20 \mathrm{~s}$ of circuit time (which is 200 periods of the input's sine function). Controls with up to $200 \mathrm{~s}$ of circuit time showed that the spectral analyses in this paper were not affected. Simulation time for $20 \mathrm{~s}$ of circuit time on a Xeon E5-1650 CPU reached several hours for the simulations with the highest noise. 
Output waveforms of the circuit were exported and signal processed in the Mathworks MATLAB software. A binary signal was first generated, which reproduced the spikes generated by the circuit: it is 1 when the neuron declares a spike, and 0 the rest of the time. We performed FFT on this binary signal, and not on an actual voltage waveform from the circuit. That way, subthreshold oscillations of the circuits cannot affect the FFT, and any spectral feature is really on the spiking pattern of the neuron.
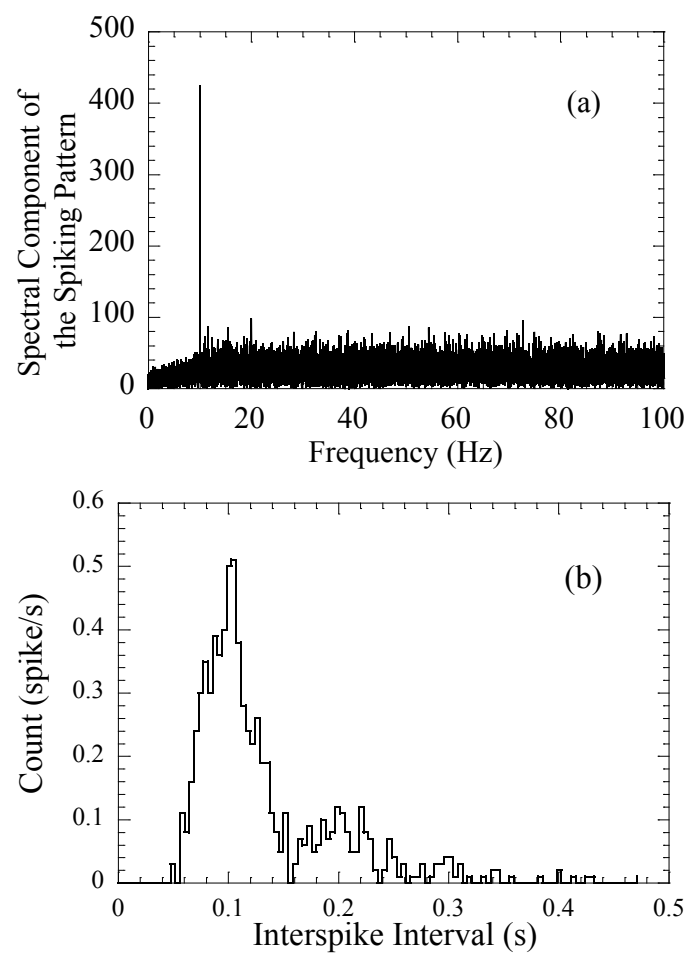

Figure 3. (a) FFT (b) Histogram of interspike intervals (both correspond to the case of Figure 2(c), input noise of $0.1 \mathrm{~V}^{2} / \mathrm{kHz}$ ).

\section{RESULTS}

Figure 2 presents the behavior of the circuit, as simulated with different noise level. We plot the input voltage with noise (superimposed with the input voltage without noise to guide the eye), as well as the "membrane current" $I_{\text {mem, which }}$ represents the internal state of the neuron. We materialize the neuron's spike by a thick vertical blue line on the time axis.

When there is no added noise (Figure 2(a)), the membrane current $\mathrm{I}_{\text {mem }}$ oscillates, but the positive feedback is never sufficient to generate a spike: the neuron has no answer. When there is moderate noise (Figure 2(b), $0.04 \mathrm{~V}^{2} / \mathrm{kHz}$ ), the membrane currents appears noisy and the positive feedback can sometimes be sufficient to generate spikes. These remain rare and seem random. Where there is higher noise (Figure $2(\mathrm{c}), 0.1 \mathrm{~V}^{2} / \mathrm{kHz}$ ), the behavior looks very different. A spike is generated almost every period of the $\mathrm{AC}$ input. Two spikes are never generated during the same AC input period. Basically, the neurons spikes in phase with the input, missing a period occasionally. It should be noted that, in this regime, there is so much noise that the sine function cannot be perceived in the input voltage. When there is even higher noise (Figure 2(d), $0.6 \mathrm{~V}^{2} / \mathrm{kHz}$ ), the neuron spikes frequently and erratically.

If we perform FFT on the firing pattern, we get a spectrum as in Figure 3(a) (which corresponds to the signal of Figure 2(c)). A clear peak is seen at $10 \mathrm{~Hz}$, the frequency of the sine function in the input voltage. We define the signal-to-noise ratio of the spiking output as the ratio of the height of this peak to the average level of the FFT on the $1-100 \mathrm{~Hz}$ range (excluding the peak). If we plot the signal-to-noise ratio as a function of the input noise levels, a bell curve is seen (Figure 4 , full line " $\mathrm{V}_{\text {ref }}=0.20 \mathrm{~V}$ "). For zero noise, there is no signal since the neuron is not answering. For high noise, the peak is decreasing (the neuron starts to spike randomly). A maximum is existing in-between. This kind of bell curve is a signature of the stochastic resonance phenomenon [11].

Similarly, in Fig 3(b), we plot a histogram of interspike interval in the situation of Fig 2(c). We see that the most likely interspike intervals are around the input's sine function period $(0.1 \mathrm{~s})$. Interspike intervals around twice and three times this period also occur, and correspond to the situation where the neuron missed an input's period. This kind of inter-event intervals curve is also a signature of stochastic resonance [11].

The results presented in Figures 2 and 3 are presented with an input's frequency of $10 \mathrm{~Hz}$. A similar bell curve and similar interspike interval histograms were obtained for input frequencies from 4 to $25 \mathrm{~Hz}$, without changing any bias or capacitance in the circuit.

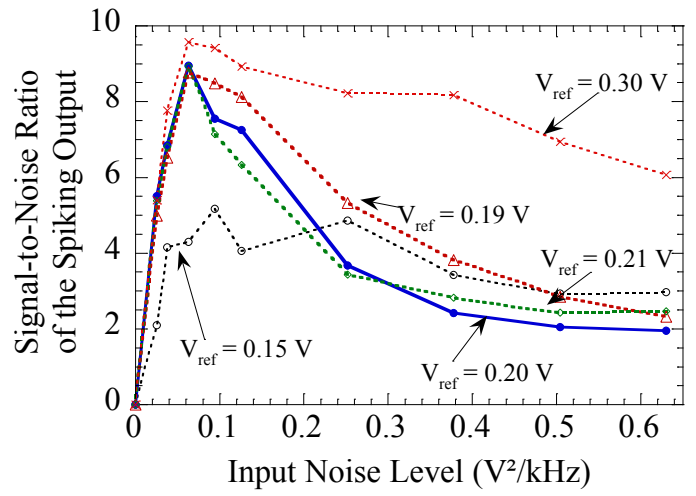

Figure 4. Height of the spectral peak at $10 \mathrm{~Hz}$ in the FFT, as a function of noise level, for different refractory periods.

\section{DISCUSSION}

Interestingly, stochastic resonance was easily obtained whatever the values of most of the biases and of the capacitors in the neuron's circuit. In particular it is obtained whatever the time constant of the low pass filter (which is determined by capacitance $\mathrm{C}_{\text {mem }}$ and bias $\mathrm{V}_{\text {leak }}$ [3]), and the values of $\mathrm{V}_{\text {thresh }}$ (provided they do not impair the circuit's basic functionality).

However, we found that the parameters regarding the refractory period played a more important role. The refractory period is the time during which a neuron is prevented from firing after it has fired. It is determined by the capacitor $\mathrm{C}_{\mathrm{ref}}$ and the bias $\mathrm{V}_{\text {ref }}$ in the circuit. The refractory period has a linear dependence with $\mathrm{C}_{\text {ref }}$ and an exponential dependence with $\mathrm{V}_{\text {ref. [3] }}$. This is well seen in Figure 4. 
For short refractory period (e.g. $\left.\mathrm{V}_{\text {ref }}=0.30 \mathrm{~V}\right)$, the neurons may spike multiple times by input period. A nice bell curve is not seen, and the interspike interval histogram is broad and does not show peaks around $0.1 \mathrm{~s}$ and $0.2 \mathrm{~s}$ like in Fig. 3(b). For long refractory period (e.g. $\mathrm{V}_{\text {ref }}=0.15 \mathrm{~V}$ ) the neurons rarely spike, since the refractory period is longer than one input's threshold. In both of these situations, the firing pattern of the neuron does not follow the input's periods and stochastic resonance cannot be useful. Real stochastic resonance, characterized by interspike interval histogram similar to Fig. 3b, is observed on a $60 \mathrm{mV}$ range of $\mathrm{V}_{\text {ref }}(0.18$ $0.24 \mathrm{~V}$ ), which corresponds approximately to a factor five on the refractory period.

\section{CONCLUSION}

In this work, we have observed by circuit simulation that a neuromorphic neuron circuit could naturally exhibit stochastic resonance. The methodology consisted in applying a small sine input (which would trigger no spike in the neuron without noise), embedded in noise to the neuron. When noise is present, the neuron spikes, and the spiking response shows a spectral response at the input's frequency. This response has a maximum for a given range of noise: the stochastic resonance. The maximum resonance is not sharp. When at this maximum, if the refractory period is appropriate, the neuron spikes in phase with the input (sometimes missing a period). This means that it turned the input, a sine function embedded in noise, into a regular spiking pattern, which may be exploited by other neuromorphic circuits directly.

Exploiting this idea could allow neuromorphic circuits to work with weak and extremely noisy signals, for example coming from inexpensive sensors, and to develop new concepts and model of computation. Future work should focus on demonstrating functions associating several neurons with the stochastic resonance property.

\section{ACKNOWLEDGMENT}

The authors would like to thank A. Bournel and P. Dollfus for helpful discussions, and C. Chassat for computer assistance.

\section{REFERENCES}

[1] J. P. Campbell, L. C. Yu, K. P. Cheung, J. Qin, J. S. Suehle, A. Oates, and K. Sheng, "Large random telegraph noise in sub-threshold operation of nano-scale nMOSFETs," in IEEE Int. Conf. IC Design and Technology (ICICDT '09), 2009, pp. 17-20.

[2] J. Zhuge, R. Wang, R. Huang, Y. Tian, L. Zhang, D.-W. Kim, D. Park, and Y. Wang, "Investigation of Low-Frequency Noise in Silicon Nanowire MOSFETs," IEEE Electron Dev. Lett., vol. 30, no. 1, pp. 57 -60 , Jan. 2009.

[3] P. Livi and G. Indiveri, "A current-mode conductance-based silicon neuron for address-event neuromorphic systems," in IEEE Int. Symp. on Circuits and Systems (ISCAS), 2009, pp. 2898-2901.

[4] J. V. Arthur and K. A. Boahen, "Silicon-Neuron Design: A Dynamical Systems Approach," IEEE Trans. Circuits Syst. Regul. Pap., vol. 58, no. 5, pp. 1034-1043, 2011.

[5] P. Lichtsteiner, C. Posch, and T. Delbruck, "A 128x 128120 dB 15 mus Latency Asynchronous Temporal Contrast Vision Sensor," IEEE J. Solid-State Circuits, vol. 43, no. 2, pp. 566-576, 2008.

[6] V. Chan, S.-C. Liu, and A. van Schaik, "AER EAR: A Matched Silicon Cochlea Pair With Address Event Representation Interface," IEEE Trans. Circuits Syst. Regul. Pap., vol. 54, no. 1, pp. 48-59, 2007.
[7] A. Longtin, "Stochastic resonance in neuron models," J. Stat. Phys., vol. 70, no. 1-2, pp. 309-327, Jan. 1993.

[8] J. K. Douglass, L. Wilkens, E. Pantazelou, and F. Moss, "Noise enhancement of information transfer in crayfish mechanoreceptors by stochastic resonance," Nature, vol. 365, no. 6444, pp. 337-340, Sep. 1993.

[9] R. Benzi, A. Sutera, and A. Vulpiani, "The mechanism of stochastic resonance," J. Phys. A-Math. Gen., vol. 14, no. 11, pp. L453-L457, Nov. 1981.

[10] K. Wiesenfeld and F. Moss, "Stochastic resonance and the benefits of noise: from ice ages to crayfish and SQUIDs," Nature, vol. 373, no. 6509, pp. 33-36, Jan. 1995.

[11] L. Gammaitoni, P. Hänggi, P. Jung, and F. Marchesoni, "Stochastic resonance," Rev. Mod. Phys., vol. 70, no. 1, p. 223, Jan. 1998.

[12] I. Y. Lee, X. Liu, B. Kosko, and C. Zhou, "Nanosignal Processing: Stochastic Resonance in Carbon Nanotubes That Detect Subthreshold Signals," Nano Lett., vol. 3, no. 12, pp. 1683-1686, Décembre 2003.

[13] T. Oya, T. Asai, and Y. Amemiya, "Stochastic resonance in an ensemble of single-electron neuromorphic devices and its application to competitive neural networks," Chaos, Solitons \& Fractals, vol. 32, no. 2, pp. 855-861, Apr. 2007.

[14] F. Hartmann, A. Forchel, I. Neri, L. Gammaitoni, and L. Worschech, "Nanowatt logic stochastic resonance in branched resonant tunneling diodes," Appl. Phys. Lett., vol. 98, no. 3, pp. 032110-032110-3, Jan. 2011.

[15] F. Hartmann, L. Gammaitoni, S. Höfling, A. Forchel, and L. Worschech, "Light-induced stochastic resonance in a nanoscale resonant-tunneling diode," Appl. Phys. Lett., vol. 98, no. 24, pp. 242109-242109-3, Jun. 2011.

[16] A. S. Samardak, A. Nogaret, N. B. Janson, A. Balanov, I. Farrer, and D. A. Ritchie, "Spiking computation and stochastic amplification in a neuron-like semiconductor microstructure," J. Appl. Phys., vol. 109, no. 10, pp. 102408-102408-5, May 2011.

[17] G. P. Harmer, B. R. Davis, and D. Abbott, "A review of stochastic resonance: circuits and measurement," IEEE Trans. Instrum. Meas., vol. 51, no. 2, pp. $299-309$, Apr. 2002

[18] D. G. Luchinsky, R. Mannella, P. V. E. McClintock, and N. G. Stocks, "Stochastic resonance in electrical circuits. I. Conventional stochastic resonance," IEEE Trans. Circuits Syst. II, vol. 46, no. 9, pp. 1205 1214, Sep. 1999.

[19] P. Gao, B. V. Benjamin, and K. Boahen, "Dynamical System Guided Mapping of Quantitative Neuronal Models Onto Neuromorphic Hardware," IEEE Trans. Circuits Syst. Regul. Pap., vol. PP, no. 99, p. $1,2012$.

[20] R. Serrano-Gotarredona, M. Oster, P. Lichtsteiner, A. LinaresBarranco, R. Paz-Vicente et al, "CAVIAR: A 45k Neuron, 5M Synapse, 12G Connects/s AER Hardware Sensory-ProcessingLearning-Actuating System for High-Speed Visual Object Recognition and Tracking," IEEE Trans. Neural Networks, vol. 20, no. 9, pp. 1417$1438,2009$.

[21] P. Merolla, J. Arthur, F. Akopyan, N. Imam, R. Manohar, and D. S. Modha, "A digital neurosynaptic core using embedded crossbar memory with $45 \mathrm{pJ}$ per spike in $45 \mathrm{~nm}, "$ in IEEE Custom Integrated Circuits Conference (CICC), 2011, pp. 1 -4.

[22] G. Indiveri, B. Linares-Barranco, T. J. Hamilton, R. EtienneCummings, T. Delbruck, S.-C. Liu, P. Häfliger, S. Renaud, J. Schemmel, G. Cauwenberghs, J. Arthur, S. Saighi, J. Wijekoon, and K. Boahen, "Neuromorphic silicon neuron circuits," Front. Neuromorphic Engineering, vol. 5, p. 73, 2011.

[23] J. Partzsch, S. Hartmann, C. G. Mayr, S. Höppner, and B. Vogginger, "VLSI implementation of a $2.8 \mathrm{Gevent} / \mathrm{s}$ packet-based AER interface with routing and event sorting functionality," Front. Neuromorphic Engineering, vol. 5, p. 117, 2011.

[24] C. Bartolozzi, S. Mitra, and G. Indiveri, "An ultra low power currentmode filter for neuromorphic systems and biomedical signal processing," in IEEE Biomedical Circuits and Systems Conf. (BioCAS 2006), 2006, pp. 130-133. 